\title{
SIMULTANEOUS PYROELECTRIC AND DILATOMETRIC STUDIES OF PHASE TRANSITIONS IN TRIGLYCINE SULPHATE AND GLYCINE POSPHITE CRYSTALS
}

\author{
B. Andriyevsky, V. Kardash
}

The Ivan Franko National University of L'viv 8 Kyrylo \& Mefodii Str., 79005 L'viv, Ukraine

\section{S. DACKo AND Z. CZaPla}

Institute of Experimental Physics, University of Wrocław

9 M. Born Sqr., 50-204 Wrocław, Poland

(Received November 3, 2000; revised version February 5, 2001)

Temperature dependences of the linear dimension $L(T)$ and spontaneous polarization $P_{\mathrm{s}}(T)$ for triglycine sulphate and glycine posphite single crystals have been measured in the same run of temperature. A decrease in the spontaneous electrostriction coefficient $Q_{L}$ of both crystals studied is displayed when approaching the corresponding phase transition point $T_{c}$. A temperature dependence of the coefficients $Q_{L}(T)$ shows that the rates of temperature changes of displacement of the atomic complexes of corresponding crystal structure forming the square of spontaneous polarization $P_{\mathrm{s}}^{2}$ (primary order parameter) and the displacements of all atomic complexes forming the spontaneous linear thermal expansion $\delta L_{\mathrm{s}} / L$ (secondary order parameter) are different. The applying external electric field $E=3 \times 10^{5} \mathrm{~V} / \mathrm{m}$ to triglycine sulphate crystal does not influence essentially the temperature dependence of $Q_{L}(T)$ in the temperature range of phase transition below $T_{c}$.

PACS numbers: 77.80 . Bh

\section{Introduction}

Critical behavior of ferroelectrics at the continuous phase transitions is often studied not only by means of the investigation of the temperature dependence of spontaneous polarization $P_{\mathrm{s}}(T)[1-4]$,

$$
P_{\mathrm{s}}(T)=A_{\mathrm{P}}\left(T_{\mathrm{c}}-T\right)^{\beta}
$$


but also using the spontaneous parts of such dependences for different physical values $Y_{\mathrm{s}}(T)$ (linear dimensions of crystal, optical birefringence, refractive index and others) [5-7]. As it was determined in the investigations of known ferroelectrics, e.g. $\mathrm{BaTiO}_{3}[2,8]$, in the crystals with a symmetry center in the paraelectric phase the quadratic relation between such the values $Y_{\mathrm{s}}(T)$ and spontaneous polarization $P_{\mathrm{s}}(T)$ in the ferroelectric phase takes place

$$
Y_{\mathrm{s}}(T)=Q_{Y} P_{\mathrm{s}}^{2}(T)=Q_{Y} A_{\mathrm{P}}^{2}\left(T_{\mathrm{c}}-T\right)^{2 \beta}=A_{Y}^{2}\left(T_{\mathrm{c}}-T\right)^{2 \beta^{\prime}} .
$$

The aim of the present investigation was to study the temperature dependences of the coefficients of spontaneous linear electrostriction $Q_{Y}=Q_{L}$ for triglycine sulphate, $\left(\mathrm{NH}_{2} \mathrm{CH}_{2} \mathrm{COOH}\right)_{3} \cdot \mathrm{H}_{2} \mathrm{SO}_{4}$ (TGS), and glycine posphite, $\left(\mathrm{NH}_{2} \mathrm{CH}_{2} \mathrm{COOH}\right) \cdot \mathrm{H}_{3} \mathrm{PO}_{3}(\mathrm{GPI})$, single crystals. Both crystals possess a symmetry center in the paraelectric phase $[8,9]$. If the coefficient $Q_{L}$ depends on temperature, then the inequality $\beta^{\prime} \neq \beta$ takes place. Taking into account that the index $\beta$ of the power-like dependence characterizes a velocity of temperature change of corresponding characteristics of crystal, the nontrivial temperature dependence of this coefficient, $Q_{L}(T) \neq$ const, could testify to different rates of temperature changes of displacement of the atomic complexes of the crystal structure forming the square of spontaneous polarization $P_{\mathrm{s}}^{2}$ and the displacements of all atomic complexes forming the spontaneous linear thermal expansion of crystal $\delta L_{\mathrm{s}} / L$. Such temperature dependences were already displayed in optical studies in TGS [10], dilatometric measurements of dimethylammonium aluminum sulphate hexahydrate (DMAAlS) [11] and GPI [12] crystals. But these results were obtained using only the reference data on temperature dependences of spontaneous polarization [8, 13-15]. In this paper the simultaneous measurements of dilatative and pyroelectric investigations of TGS and GPI crystals were conducted to verify the conclusion about temperature dependences of spontaneous electrostriction effect obtained in the papers [10-12].

\section{Experimental}

Simultaneous measurements of temperature dependences of pyroelectric signal and linear dimension of crystalline samples of TGS and GPI (Fig. 1) were con-
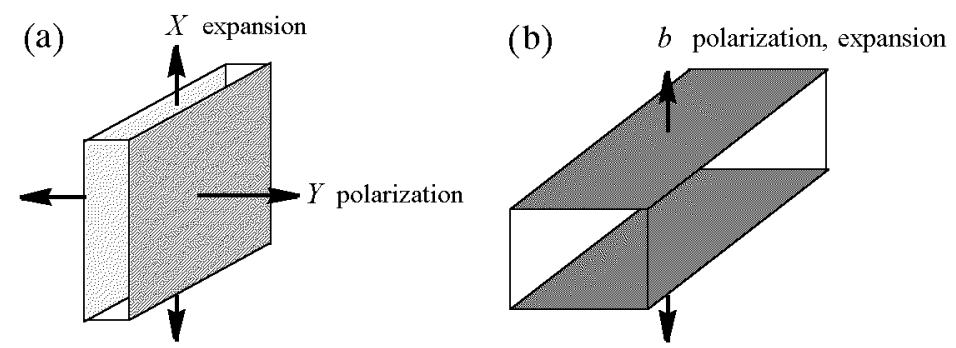

Fig. 1. Directions of measuring the polarization signal and linear expansion of TGS (a) and GPI (b) samples. 
ducted using the standard equipment for piroelectric measurement and the quartz dilatometer of capacitor type [11, 12] with a precision LCR-meter HP 4284A. Conducting liquid silver paste was deposited on two opposite ferroelectric sides of plate parallel crystalline sample of the typical dimensions of $10 \times 7 \times 2 \mathrm{~mm}^{3}$ (Fig. 1). The temperature in the chamber was controlled with an accuracy not worse than $5 \times 10^{-3} \mathrm{~K}$. One magnitude on cooling run $1 \mathrm{~K} / \mathrm{min}$ was measured in experiments.

\section{Results and discussion}

For both crystals the square of polarization $P_{\mathrm{s}}^{2}$ and the corresponding relative spontaneous expansion $\delta L_{\mathrm{s}} / L$ do not depend linearly upon temperature $T$, and character of these dependences corresponds to different magnitudes of the indices $\beta_{P s}=\beta$ and $\beta_{\delta L s}=\beta^{\prime}$ of the power-like dependences (1) and (2):

$$
\beta<\beta^{\prime}<0.5 \text {. }
$$

On the basis of this relation one can suggest that in polar phase of TGS crystal the rates of temperature changes of displacement of the atomic complexes of the crystal structure forming the square of spontaneous polarization $P_{\mathrm{s}}^{2}$ and the displacements of all atomic complexes forming the spontaneous linear thermal expansion of crystal $\delta L_{\mathrm{s}} / L$ are different.

Taking into account that the relative spontaneous expansion $\delta L_{\mathrm{s}} / L$ is proportional to the square of spontaneous polarization $P_{\mathrm{s}}^{2}$,

$$
\delta L_{x \mathrm{~s}} / L_{x}=Q_{L x} P_{\mathrm{s}}^{2},
$$

we have calculated the coefficients of spontaneous electrostriction $Q_{L}$, for TGS and GPI crystals (Figs. 2,3). In both cases the coefficient $Q_{L}$ essentially decreases when approaching the corresponding phase transition temperatures $T_{\mathrm{c}}$ in the fer-

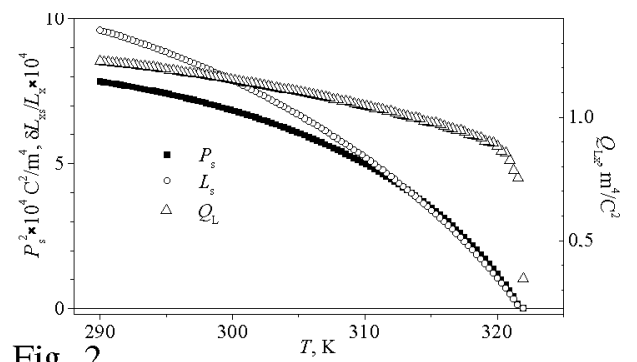

Fig. 2

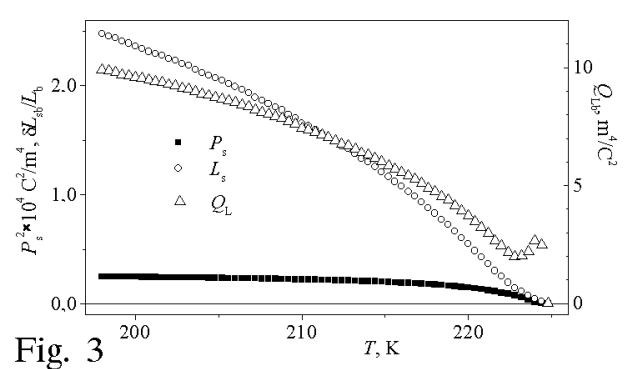

Fig. 3

Fig. 2. Temperature dependences of the square of spontaneous polarization $P_{\mathrm{s}}{ }^{2}(T)$, the relative spontaneous expansion $\delta L_{5 x} / L_{x}$ along the crystal physical $x$-direction and the corresponding coefficient of spontaneous linear electrostriction $Q_{L x}(T)$ for TGS crystal. Fig. 3. Temperature dependences of the square of spontaneous polarization $P_{\mathrm{s}}^{2}(T)$, the relative spontaneous expansion $\delta L_{\mathrm{s} b} / L_{b}$ along the same $b$-direction of spontaneous polarization and the corresponding coefficient of spontaneous linear electrostriction $Q_{L b}(T)$ for GPI crystal. 
roelectric phase. In a relatively wide temperature range of the ferroelectric phase of TGS crystal, $290-320 \mathrm{~K}$, this decrease is approximately $20 \%$ of the magnitude $Q_{L}$ at the initial temperature $T=290 \mathrm{~K}:\left\{Q_{L}(290)-Q_{L}(320)\right\} / Q_{L}(290)=0.2$. The greatest decrease in $Q_{L}(\sim 50 \%)$ takes place in the narrow temperature range of $320-323 \mathrm{~K}$ in the vicinity of the phase transition point $T_{c}=323 \mathrm{~K}$ (Fig. 2 ). The magnitude of the electrostriction coefficient $Q_{L x}$ of TGS crystal for room temperature obtained, $Q_{L x} \approx 1.2 \mathrm{~m}^{4} / \mathrm{C}^{2}$, agrees well with the data of [16].

In contrast to TGS crystals, the main decrease in $Q_{L b}$ coefficient of approximately $80 \%$ for GPI one takes place in the wide temperature range of 198-223 K (Fig. 3). This difference can be connected with the fact that the phase transition in GPI is placed on the corresponding phase diagram closer to the tricritical point than the one in TGS crystal [14]. As the result GPI shows a high hydrostatic pressure sensitivity of the PT point $T_{c}$, four times greater than the one for TGS [17]. Analogous to GPI behavior of temperature dependence of the coefficient $Q_{L}(T)$ is characteristic of the ferroelectric phase of DMAAIS crystal [11], which is also close to the tricritical point at the normal conditions.

A decrease in the coefficient $Q_{L}$ when approaching the phase transition points $T_{\mathrm{C}}$ of proper ferroelectrics TGS and GPI in polar phases confirms the above formulated suggestion relating to the nonequality (3) and shows that the difference, between the rates of temperature changes of displacement of the atomic complexes of the crystal structure forming the square of spontaneous polarization $P_{\mathrm{s}}^{2}$ (primary order parameter) and the displacements of all atomic complexes forming the spontaneous linear thermal expansion of crystal $\delta L_{\mathrm{s}} / L$ (secondary order parameter), is evidently inverse proportional to the temperature separation from the corresponding phase transition points $\left(T_{\mathrm{c}}-T\right)$.

To study an influence of external electric field on the temperature dependence of coefficients of spontaneous electrostriction we have conducted analogous measurements for TGS crystals by means of applying the external electric field $E=3 \times 10^{5} \mathrm{~V} / \mathrm{m}$ along the polar $y$-axes. An influence of this field changes essentially the forms of temperature dependences of $P_{\mathrm{s}}^{2}(T)$ and $\delta L_{\mathrm{s} x} / L_{x}=f(T)$ (Figs. 4, 5). These dependences become more close to the linear one on temperature, more smooth in the region of the phase transition point (Figs. 4, 5). In the range of $300-320 \mathrm{~K}$ the coefficient of spontaneous electrostriction $Q_{L}$ slightly increases $(\sim 5 \%)$ and decreases in the range of $320-325 \mathrm{~K}(\sim 10 \%)$ (Fig. 6$)$. Thus a decrease in the coefficient of spontaneous electrostriction $Q_{L}$ in the range of 320-325 K close to the phase transition point of TGS crystal can be regarded as a stable peculiarity, not depending upon the presence of external electric field $E=3 \times 10^{5} \mathrm{~V} / \mathrm{m}$. In the case of external electric field, the indices $\beta_{E}$ and $\beta_{E}^{\prime}$ of the power-like temperature dependences (2) become intermediate between the corresponding magnitudes without external electric field and the magnitude of 0.5 : $\beta<\beta_{E}<0.5$.

To determine the temperature dependence of polarization current $I_{\mathrm{p}}(T)$ and then the temperature dependence of spontaneous polarization $P_{\mathrm{s}}(T)$ we have used the known fact that in a pyroelectric measurement the sign of polarization current $I_{\mathrm{p}}$ one-to-one corresponds upon the sign of change of temperature $\mathrm{d} T$. When temperature increases $(\mathrm{d} T>0)$ the electric current $I_{\mathrm{i}}$ measured can be written in 

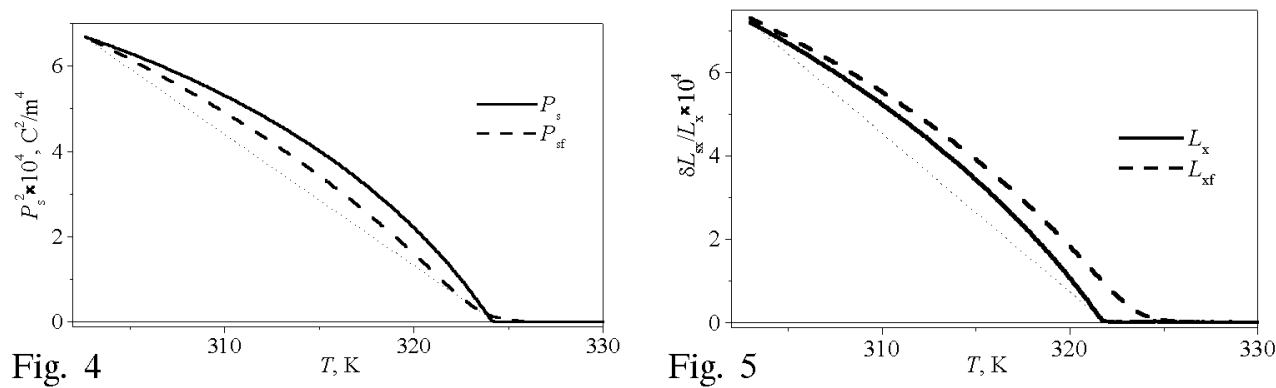

Fig. 4. Temperature dependences of the square of spontaneous polarization $P_{\mathrm{s}}^{2}(T)$ for electrically free sample of TGS crystal (solid line) and for sample with the applying external electric field $E=3 \times 10^{5} \mathrm{~V} / \mathrm{m}$ along the $y$-direction of spontaneous polarization (dashed line).

Fig. 5. Temperature dependences of the relative spontaneous expansion $\delta L_{\mathrm{s} x} / L_{x}$ along the crystal physical $x$-direction for electrically free sample of TGS crystal (solid line) and for sample with the applying external electric field $E=3 \times 10^{5} \mathrm{~V} / \mathrm{m}$ along the $y$-direction of spontaneous polarization (dashed line).

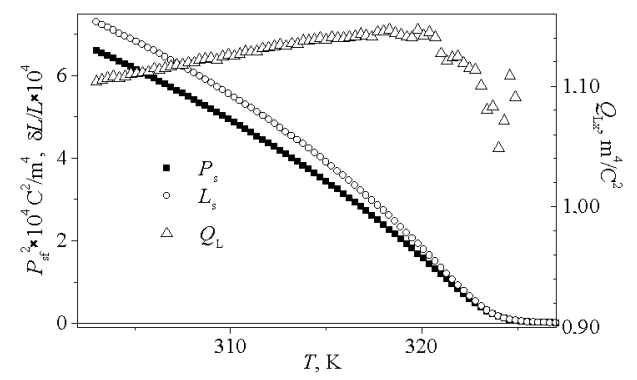

Fig. 6. Temperature dependences of the square of spontaneous polarization $P_{\mathrm{s}}^{2}(T)$, the relative spontaneous expansion $\delta L_{\varepsilon x} / L_{x}$ along the crystal physical $x$-direction and the corresponding coefficient of spontaneous linear electrostriction $Q_{L x}(T)$ for TGS crystal with the applying external electric field $E=3 \times 10^{5} \mathrm{~V} / \mathrm{m}$ along the $y$-direction of spontaneous polarization.

the following form:

$$
I_{\mathrm{i}}=I_{\mathrm{c}}-I_{\mathrm{p}},
$$

where $I_{\mathrm{C}}$ is the conductivity current and $I_{\mathrm{p}}$ is the polarization one. In the case of a decrease in temperature $(\mathrm{d} T<0)$ the relation for the electric current $I_{\mathrm{d}}$ measured takes another form:

$$
I_{\mathrm{d}}=I_{\mathrm{c}}+I_{\mathrm{p}} .
$$

From Eqs. (5) and (6) one can obtain easily the relations for the conductivity $I_{\mathrm{C}}$ and polarization $I_{\mathrm{p}}$ currents

$$
I_{\mathrm{c}}=\left(I_{\mathrm{i}}+I_{\mathrm{d}}\right) / 2, \quad I_{\mathrm{p}}=\left(I_{\mathrm{d}}-I_{\mathrm{i}}\right) / 2 .
$$




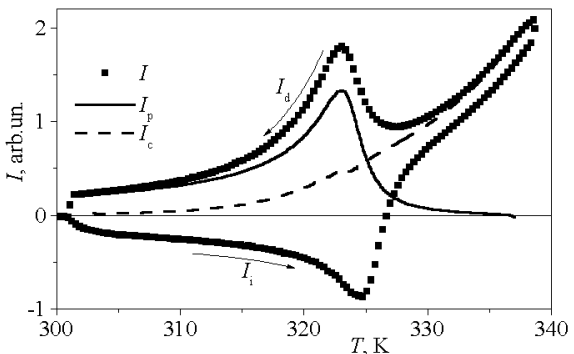

Fig. 7

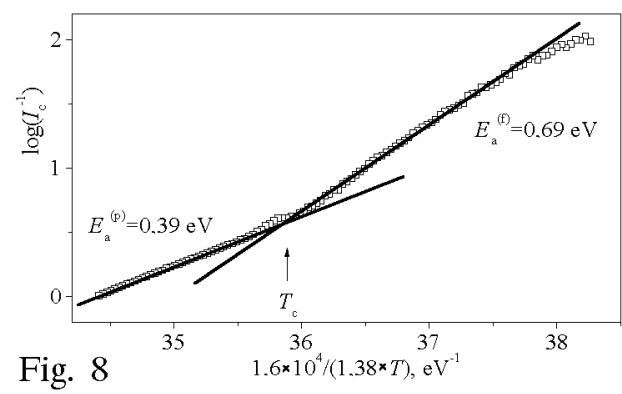

Fig. 8

Fig. 7. Temperature dependences of the current $I(T)$ measured for $y$-direction of spontaneous polarization of TGS crystal for the increase $\left(I_{\mathrm{i}}\right)$ and decrease $\left(I_{\mathrm{d}}\right)$ of temperature with applying external electric field $E=3 \times 10^{5} \mathrm{~V} / \mathrm{m}$ along the $y$-direction: $I_{\mathrm{p}}$ and $I_{\mathrm{c}}$ are the polarization and conductivity components of $I$ current, respectively.

Fig. 8. Dependence of logarithm of the inverse conductivity current $\log \left(I_{c}^{-1}\right)$ upon the inverse temperature $T^{-1}$ for the $y$-direction of spontaneous polarization of TGS crystal with the applying external electric field $E=3 \times 10^{5} \mathrm{~V} / \mathrm{m}$ along the $y$-direction.

The corresponding results for TGS crystal are shown in Fig. 7. An extreme like behavior of the dependences $I(T)$ and $I_{\mathrm{p}}(T)$ are caused by the fast temperature change of spontaneous polarization $P_{\mathrm{s}}$ near the phase transition temperature $T_{\mathrm{c}}$. Because of a slight relation between electric conductivity and spontaneous polarization of the crystal the temperature dependence of conductivity current $I_{c}(T)$ does not display visibly any anomaly at $T_{\mathrm{c}}$. But presenting the temperature dependence of conductivity current $I_{\mathrm{c}}(T)$ in the coordinates $\log \left(I_{\mathrm{c}}^{-1}\right)-(k T)^{-1}$ according to the known exponential form

$$
I_{\mathrm{c}}(T) \sim \exp \left(-E_{\mathrm{a}} / k T\right),
$$

one can see the distinct break at the phase transition point of TGS crystal (Fig. 8). Thus, the paraelectric and ferroelectric phases of TGS crystals are characterized by different activation energies of electric conductivity: $E_{\mathrm{a}}^{(\mathrm{p})}=0.39 \mathrm{eV}$ and $E_{\mathrm{a}}^{(\mathrm{f})}=0.69 \mathrm{eV}$. This is a result of the considerable reconstruction of extrinsic energy states of TGS crystal caused by the structural reconstruction at the phase transition. The corresponding charge carriers can be as extrinsic particles or defects of the main crystal structure. The high temperature paraelectric phase of TGS crystal is characterized by a smaller activation energy $E_{\mathrm{a}}$ of extrinsic conductivity, whereas the deeper traps for the corresponding carriers are characteristic of the low temperature ferroelectric phase.

\section{Conclusions}

1. A decrease in the spontaneous electrostriction coefficient $Q_{L}$ of the proper ferroelectric crystals TGS and GPI is displayed when approaching the corresponding phase transition point $T_{\mathrm{C}}$.

2. The temperature dependence of the spontaneous electrostriction coefficient $Q_{L}(T)$ of TGS and GPI crystals shows that the rates of temperature changes 
of displacement of the atomic complexes of the corresponding crystal structure forming the square of spontaneous polarization $P_{\mathrm{s}}^{2}$ (primary order parameter) and the displacements of all atomic complexes forming the spontaneous linear thermal expansion $\delta L_{\mathrm{s}} / L$ (secondary order parameter) are different. This difference is evidently inverse proportional to the temperature separation from the phase transition point $\left(T_{\mathrm{c}}-T\right)$.

3. The main temperature change of the spontaneous electrostriction coefficient $Q_{L}$ of TGS crystals takes place in the relatively narrow range below $T_{c}$, $\Delta T=T_{\mathrm{c}}-T \approx 3 \mathrm{~K}$, whereas for GPI crystal this change takes place in a much wider range, $\Delta T \approx 20 \mathrm{~K}$. This result can be connected with the fact that the phase transition in GPI is closer to the tricritical one than that in TGS crystal.

4. The applying external electric field $E=3 \times 10^{5} \mathrm{~V} / \mathrm{m}$ to TGS crystal does not influence essentially the decrease in the coefficient of spontaneous electrostriction $Q_{L}$ in the temperature range of $320-325 \mathrm{~K}$ below $T_{\mathrm{c}}$. In this case the indices $\beta_{E}$ and $\beta_{E}^{\prime}$ of the power-like temperature dependences (2) become greater than the analogous ones without external electric field, but staying smaller than 0.5 .

5. The high temperature paraelectric phase of TGS crystal is characterized by the activation energy of extrinsic conductivity, $E_{\mathrm{a}}^{(\mathrm{p})}=0.39 \mathrm{eV}$, smaller than the one for the low temperature ferroelectric phase, $E_{\mathrm{a}}^{(\mathrm{f})}=0.69 \mathrm{eV}$.

\section{References}

[1] S. Ma, Modern Theory of Critical Phenomena, London 1976, p. 298.

[2] M.E. Lines, A.M. Glass, Principles and Application of Ferroelectrics and Related Materials, Clarendon Press, Oxford 1977, p. 736.

[3] M.K. Gergs, D. Michel, H. Schlemmbach, W. Windsch, J. Phys. C, Solid State Phys. 19, 2431 (1986).

[4] R. Cach, Dielectric Non-Linear Properties of some Real Ferroelectric Crystals, Wydawnictwo Uniwersytetu Wrocławskiego, Wrocław 1992, p. 69.

[5] A.S. Sonin, A.S. Vasilevskaya, Electrooptical Crystals, Atomizdat, Moscow 1971 (in Russian).

[6] L.G. Lomova, A.S. Sonin, T.A. Regulskaya, Kristallografiya 13, 90 (1968) (in Russian).

[7] B. Kosturek, J. Baran, Ferroelectr. Lett. 27, 11 (2000).

[8] F. Jona, G. Shirane, Ferroelectrical Crystals, Macmillan, New York 1962.

[9] M.Th. Averbuch-Pouchot, Acta Crystallogr. C 49, 85 (1993).

[10] B.V. Andriyevsky, O.Ya. Myshchyshyn, V.Yu. Kurlyak, N.N. Romanyuk, Sov. Phys.-Crystallogr. 44, 516 (1999).

[11] D. Podsiadła, Z. Czapla, B. Andriyevsky, O. Myshchyshyn, Acta Phys. Pol. A 96, 409 (1999).

[12] B. Andriyevsky, Z. Czapla, N. Romanyuk, O. Myshchyshyn, Phys. Status Solidi A $177,575(2000)$ 
[13] R. Cach, S. Dacko, Z. Czapla, Phys. Status Solidi A 116, 827 (1989).

[14] S. Dacko, Z. Czapla, J. Baran, M. Drozd, Phys. Lett. A 223, 217 (1996).

[15] R. Tchukvinskyi, R. Cach, Z. Czapla, S. Dacko, Phys. Status Solidi A 165, 309 (1998).

[16] K.-H. Ehses, Ferroelectrics 25, 573 (1980).

[17] N. Yasuda, T. Sakurai, Z. Czapla, J. Phys., Condens. Matter 9, L347 (1997). 